\title{
Pumping Paint More Gently
}

\author{
A new range of electric piston pumps reduces resource \\ consumption in paint circulation systems and makes \\ the process more efficient. This leads to lower costs and \\ a longer service life for the pumps.
}

\begin{abstract}
Manufacturers and users of modern paint pumps have to meet increasingly high standards. As a result, more effective and more energy-efficient concepts are needed. Timmer has developed a new range of electric piston pumps (the KPE series) which can improve the efficiency of paint supply systems.

In many paint mixing rooms, there is little space available. The compact design of the new piston pumps means that they can be easily integrated with very little installation work. The pumps from the new range can also be retrofitted without problems in existing paint shops and they bring benefits for brownfield and greenfield systems.
\end{abstract}

\section{A longer service life}

The drive kinematics of the pumps and the vertical piston arrangement with integrated gravity-operated medium valves ensure that the pistons, valves and sealings are exposed to symmetric load, which significantly increases the service life of the pumps.

In addition, special ceramic-coated pistons are used, which have high-quality guiding seals. The highly durable bellows separate the paint compartment and the drive system hermetically and have an additional backup seal. This also helps to lengthen the working life of the pumps and reduce repair costs and downtimes.

The fluid transfer system has been improved to ensure that the paint is pumped gently and with reduced pulsation. The short pipes make cleaning easy and keep the consumption of cleaning agent to a minimum. Together with the small volume of paint in the fluid unit, this also reduces the environmental impact of the entire system.

A new range of electric piston pumps will help to improve paint supply systems.

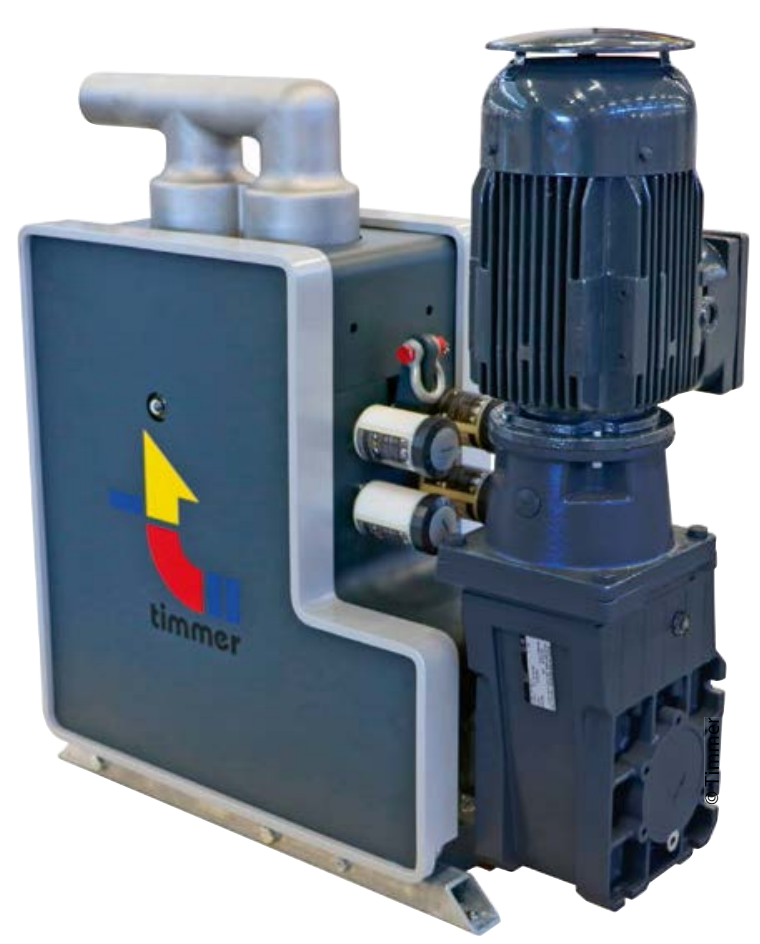

\section{Simpler maintenance}

The automated lubricant supply ensures that the bearings in the drive system are permanently lubricated. A reliable supply of fresh lubricant is automatically provided and there is no need for manual greasing.

In order to reduce production downtimes significantly, the maintenance concept, known as "service on-the-spot", has a modular structure that meets the needs of users and operators. This means that the pumps do not have to be removed from the paint supply system, which can be complicated and time-consuming. They can be maintained easily and quickly from above, without the need to disconnect them. The option is also available of replacing the entire paint unit to keep production downtime to a minimum.

\section{Customised paint supply system}

The new pumps are significantly cheaper to run, use less energy and provide higher levels of performance when compared with conventional pneumatic pumps for paint supply systems in large and medium-sized paint shops. Because of their increased pumping capacity in long-term operation, smaller pump models can often be used. By minimising the pressure and speed of the paint outside production periods, the paint supply can be adjusted to meet users' actual needs. This means that the paint is pumped more gently and the service life of the pumps is extended.

The following versions of the pumps are currently available: $30 \mathrm{l} / \mathrm{min}$. (KPE1030), $40 \mathrm{l} / \mathrm{min}$. (KPE1040) and $60 \mathrm{l} / \mathrm{min}$. (KPE1060). //

\section{Contact}

\section{Timmer GmbH}

Neuenkirchen

Germany

Gerd Lübberink, Export Sales Manager

sales@timmer.de

www.timmer-pumps.com 